\title{
OPEN Distant metastasis time to event analysis with CNNs in independent head and neck cancer cohorts
}

\author{
Elia Lombardo ${ }^{1,2}$, Christopher Kurz ${ }^{1,2}$, Sebastian Marschner ${ }^{1}$, Michele Avanzo4, \\ Vito Gagliardi ${ }^{4}$, Giuseppe Fanetti ${ }^{5}$, Giovanni Franchin ${ }^{5}$, Joseph Stancanello ${ }^{6}$, \\ Stefanie Corradini ${ }^{1}$, Maximilian Niyazi ${ }^{1}$, Claus Belka ${ }^{1,3}$, Katia Parodi ${ }^{2}$, Marco Riboldi ${ }^{2,7}$ \& \\ Guillaume Landry ${ }^{1,2,7 凶}$
}

Deep learning models based on medical images play an increasingly important role for cancer outcome prediction. The standard approach involves usage of convolutional neural networks (CNNs) to automatically extract relevant features from the patient's image and perform a binary classification of the occurrence of a given clinical endpoint. In this work, a 2D-CNN and a 3D-CNN for the binary classification of distant metastasis (DM) occurrence in head and neck cancer patients were extended to perform time-to-event analysis. The newly built CNNs incorporate censoring information and output DM-free probability curves as a function of time for every patient. In total, 1037 patients were used to build and assess the performance of the time-to-event model. Training and validation was based on 294 patients also used in a previous benchmark classification study while for testing 743 patients from three independent cohorts were used. The best network could reproduce the good results from 3 -fold cross validation [Harrell's concordance indices ( $\mathrm{HCls}$ ) of $0.78,0.74$ and 0.80 ] in two out of three testing cohorts ( $\mathrm{HCls}$ of $0.88,0.67$ and 0.77 ). Additionally, the capability of the models for patient stratification into high and low-risk groups was investigated, the CNNs being able to significantly stratify all three testing cohorts. Results suggest that image-based deep learning models show good reliability for DM time-to-event analysis and could be used for treatment personalisation.

Biology-driven personalised treatment is a landmark in the development of precision radiation oncology. Over the past years, several biomarkers (e.g. human papilloma virus (HPV) status or positron emission tomography based hypoxia levels) have been proposed to help clinical decision making for improved management of certain cancers ${ }^{1}$.

Radiomics relies on non-invasive biomarkers based on advanced imaging analytics ${ }^{2}$ and has been shown to be able to unravel tumor phenotype in multiple studies ${ }^{3-6}$. The typical radiomics workflow involves imaging of the patient, identification of the gross tumor volume (GTV), conversion of images to higher dimensional data (i.e. radiomic features) and the subsequent integration and mining of these data for model building. Thus, radiomics allows to build diagnostic, prognostic and predictive models for clinical outcomes using imaging data which is acquired as a part of clinical routine ${ }^{7}$. However, several challenges have to be faced during radiomic signature development such as issues in reproducibility, standardisation in both the image acquisition, the handcrafted feature extraction and the statistical model building, and other limitations and pitfalls ${ }^{8}$. For instance, Welch et al. ${ }^{9}$ have shown that a previously developed set of radiomic features was a surrogate for tumor volume, highlighting the need for simple baseline models to be compared with advanced radiomic signatures.

Conventional radiomic models are built using machine learning algorithms: for binary classification tasks (e.g. whether the patient survives or not) typically random forests, support vector machines or artificial neural networks (ANNs) are used, while for survival analysis (i.e. predicting time-to-events or risks for clinical outcomes) Cox proportional hazards regression, random survival forests, and support vector survival methods are commonly used ${ }^{10}$. In the past few years, a sub-field of machine learning called deep learning ${ }^{11}$ has been widely

\footnotetext{
${ }^{1}$ Department of Radiation Oncology, University Hospital, LMU Munich, Munich 81377, Germany. ${ }^{2}$ Department of Medical Physics, Faculty of Physics, Ludwig-Maximilians-Universität München, Garching 85748, Germany. ${ }^{3}$ German Cancer Consortium (DKTK), Munich 81377, Germany. ${ }^{4}$ Medical Physics Department, Centro di Riferimento Oncologico di Aviano (CRO) IRCCS, 33081 Aviano, Italy. ${ }^{5}$ Radiation Oncology Department, Centro di Riferimento Oncologico di Aviano (CRO) IRCCS, 33081 Aviano, Italy. ${ }^{6}$ Guerbet SA, Villepinte, France. ${ }^{7}$ These authors contributed equally: Marco Riboldi and Guillaume Landry. ${ }^{\circledR}$ email: guillaume.landry@med.uni-muenchen.de
} 


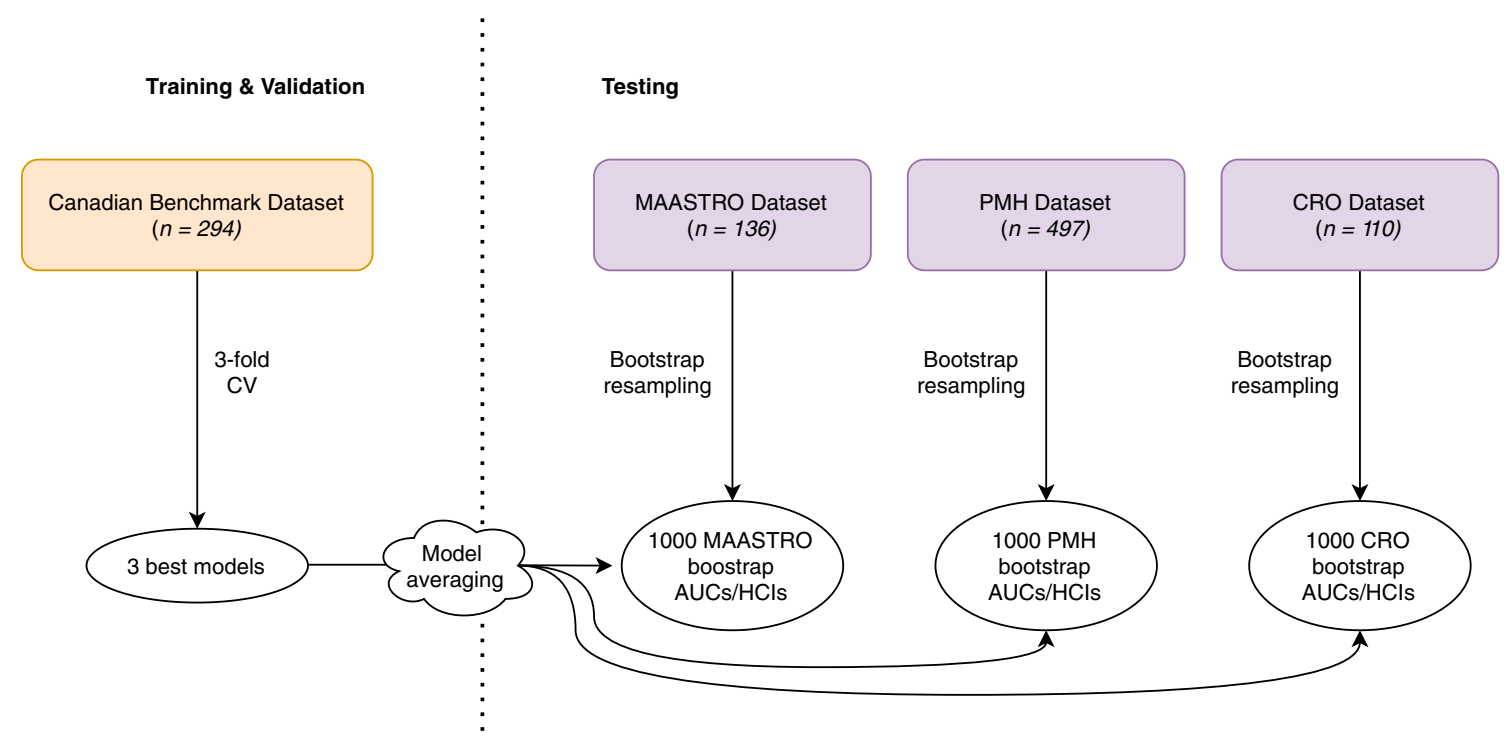

Figure 1. Cohorts training, validation and testing subdivision and analysis workflow. Firstly, the Canadian dataset used by Diamant et al. ${ }^{22}$ was used for 3-fold CV to find the hyper-parameters leading to the 3 best validation models. Then, the best validation models were applied to 1000 bootstrap samples of each of the 3 independent testing sets. The predictions of the 3 validation models for each bootstrap sample were averaged to obtain one model averaged prediction per bootstrap replicate. Prediction performance on one cohort was evaluated in terms of area under the curve (AUC) for binary classification and Harrell's concordance index (HCI) for time-to-event analysis.

and successfully adopted in a variety of fields. For medical applications, convolutional neural networks (CNNs) have been extensively used as they take spatial information into account and are therefore the preferred architecture for image-related tasks ${ }^{12}$. Compared to traditional radiomics, deep learning based radiomics approaches exploit the inherent non-linearity of deep neural networks to learn relevant features automatically ${ }^{13}$. This enables end-to-end analysis as the contoured or cropped images are given to the algorithm which outputs directly the predictions, therefore skipping the step of handcrafted feature extraction and the efforts connected with it.

Deep learning based models have been proven successful in a wide range of medical applications including classification of skin cancer $^{14}$ or extranodal extensions in head and neck squamous cell carcinoma ${ }^{15}$, fully-automated localisation and segmentation of rectal cancer ${ }^{16}$ and mortality risk stratification for lung cancer patients ${ }^{17}$ to name a few. Within radiology and radiotherapy, examples are detection of mammographic lesions ${ }^{18}$, conebeam computed tomography (CT) intensity correction ${ }^{19}$ or synthetic CT generation from magnetic resonance images $^{20,21}$. Also they have been shown to either equal or outperform their engineered features counterparts in many classification tasks. For instance Diamant et al. ${ }^{22}$ could show that a CNN trained from scratch on pretreatment CT images of head and neck cancer patients outperformed a traditional radiomics model developed in a previous study ${ }^{5}$ using exactly the same CT images. In both studies distant metastasis (DM), loco-regional failure and overall survival were used as endpoints for 300 patients available on the cancer imaging archive (TCIA) ${ }^{23,24}$ and coming from four different hospitals in Quebec, Canada. Compared to dichotomised classification, only few deep learning based survival analysis models have been applied to the field of medical imaging, although several models have already been proposed ${ }^{25-27}$. Katzman et al. ${ }^{26}$ implemented DeepSurv, a deep neural network which outputs a single number, that is, the log-risk function of a Cox proportional hazards model allowing for personalized treatment recommendations. Gensheimer et al. ${ }^{27}$ proposed Nnet-survival, a scalable discrete-time survival model for neural networks capable of outputting survival curves for every patient in a given time span.

Head and neck cancers are a set of very heterogeneous malignancies which are diagnosed worldwide more than 830,000 times and lead to more than 430,000 deaths every year ${ }^{28}$. Prognosis of these cancers depends on several factors including tumour site, TNM-stage, extracapsular nodal extension and HPV status, with 5-year survival rates that have been shown to vary from $90 \%$ for $\mathrm{HPV}^{-}$early-stage tumors, to $80-87 \%$ for $\mathrm{HPV}^{+}$and $37-58 \%$ for $\mathrm{HPV}^{-}$tumours presenting cervical lymph node metastasis ${ }^{29}$. Therefore, models that are able to identify high and low risk patients and that can support clinical decision making are highly desirable and could for instance be used for dose de-escalation in radiotherapy, with the potential of decreasing long-term toxicity.

With this work we wanted to assess the ability of CNN based approaches to accurately predict the occurrence of DM on several independent testing cohorts. To do so, we first downloaded the same 300 patients available on TCIA $^{23}$ and replicated the results obtained by Diamant et al. on this cohort using cross validation (CV) on 294 patients with a $2 \mathrm{D}-\mathrm{CNN}$. Additionally, we performed CV with a $3 \mathrm{D}-\mathrm{CNN}$, in order to exploit the full volumetric information of the tumour. Then, we downloaded two additional head and neck cancer cohorts from TCIA, 136 patients from MAASTRO clinic in the Netherland $s^{30}$ and 497 patients from Princess Margaret Cancer Centre $(\mathrm{PMH})$ in $\mathrm{Canada}^{31}$ and obtained an additional cohort of 110 patients from Centro di Riferimento Oncologico (CRO) in Italy as a third independent testing cohort. This allowed us to perform a retrospective multicentric study with 1037 CTs of different head and neck cancer patients in total. An overview of the workflow and the cohort subdivision for training, validation and testing is shown in Figure 1. 


\begin{tabular}{|l|l|l|l|l|l|}
\hline Model & CV diamant $^{22}$ & 3-fold CV & Test MAASTRO & Test PMH & Test CRO \\
\hline 2D-CNN & $0.80-0.88$ & $0.75 ; 0.83 ; 0.67$ & $0.81(0.73-0.89)$ & $0.62(0.57-0.67)$ & $0.80(0.68-0.89)$ \\
\hline 2D-CNN+Clinical & - & $0.86 ; 0.74 ; 0.80$ & $0.89(0.83-0.94)$ & $0.66(0.61-0.71)$ & $0.71(0.56-0.83)$ \\
\hline 3D-CNN & - & $0.83 ; 0.77 ; 0.79$ & $0.82(0.74-0.90)$ & $0.63(0.58-0.68)$ & $0.65(0.47-0.82)$ \\
\hline 3D-CNN+Clinical & - & $0.82 ; 0.80 ; 0.90$ & $0.87(0.80-0.94)$ & $0.66(0.61-0.71)$ & $0.67(0.55-0.79)$ \\
\hline ANN & - & $0.83 ; 0.73 ; 0.86$ & $0.86(0.79-0.92)$ & $0.66(0.61-0.71)$ & $0.74(0.62-0.84)$ \\
\hline
\end{tabular}

Table 1. Comparison of AUCs for CV in Diamant's study ${ }^{22}$ and CV and testing in our study. The numbers shown for the 3-fold CV are the AUCs obtained on the CV subsets. Numbers for the testing sets represent the median AUC and in brackets the $83 \%$ confidence intervals obtained via bootstrap re-sampling. Note that the AUCs in columns 2 and 3 can be compared directly as the CV was performed on the same cohort ${ }^{23}$.

Additionally, we extended the task of the network from binary classification to time-to-event analysis as no CNN being able to predict DM risk was found in literature. By combining Diamant's network in 2D and 3D with the survival model by Gensheimer et al. ${ }^{27}$ we have therefore constructed a deep learning framework which incorporates censoring information and is capable to output in an end-to-end fashion DM-free probability curves as function of time for every patient given the contoured pre-treatment CT image of the corresponding patient. Throughout this study, we also compared the performance of the CNNs with the performance of an ANN based on multiple clinical variables such as overall TNM stage, tumor site and volume to assess whether the CNN outperforms more classical prognostic models when it comes to discriminative performance or patient stratification. We also combined the image based CNNs with the clinical covariates based ANN in what we call $\mathrm{CNN}+\mathrm{Clinical}$, to see if a combined input could improve overall performance. Finally, to gain a better understanding of the importance of texture for the $\mathrm{CNN}$ prediction we performed a binary masking experiment with the input GTV and compared the performance on the testing sets with the performance achieved when using the standard image input.

\section{Results}

Benchmark study comparison. To verify that a CNN similar to the one built in the benchmark study ${ }^{22}$ is able to transfer its predictive performance to independent testing sets we first had to replicate the results obtained for the validation. For this, we built a $2 \mathrm{D}-\mathrm{CNN}$ as well as $3 \mathrm{D}-\mathrm{CNN}$ for classification and performed 3-fold CV on the same patient cohort used in the benchmark study. In general, the area under the curves (AUCs) obtained with the CNNs were smaller or in line with the ones obtained by Diamant et al. (see Table 1). We also performed 3-fold CV with an ANN based on clinical variables and obtained CV AUCs which were higher than for our $2 \mathrm{D}-\mathrm{CNN}$ and comparable to the ones of the 3D-CNN. For the combined CNN+Clinical models, we achieved validation AUCs which were on average higher than for the CNNs alone.

Adding independent testing cohorts. Without changing any hyper-parameter we applied the three best models from validation on a large number of patients never seen by the networks and coming from different institutions than the train and validation cohort, i.e. on three independent testing sets (Fig. 1). To be able to statistically compare results among different cohorts we performed bootstrap re-sampling on all the testing cohorts. The median AUC with 83\% confidence interval resulting from bootstrapping (see "Statistical analysis" subsection under "Methods" for the reason behind using $83 \%$ confidence) is given for each testing cohort in Table 1. The model which most consistently transferred good validation into independent testing was the $2 \mathrm{D}-\mathrm{CNN}$, being able to achieve good AUCs of around 0.8 for two out of three testing cohorts, the PMH cohort being statistically worse than the other two, with a median AUC of 0.62 . The 3D-CNN, the $2 \mathrm{D}$ and 3D CNN+Clinical and the ANN achieved very good results on the MAASTRO cohort, the other two cohorts being worse. In general, models were always able to transfer good validation prediction performance to the MAASTRO testing cohort, while for the CRO cohort the performance depended on the architecture. For the PMH cohort no network architecture yielded a median AUC above 0.66 .

Performing time-to-event analysis. Several authors ${ }^{25-27}$ have underlined the issue of censored data when performing binary outcome classification and have proposed extensions to deep learning models that are able to incorporate time-to-event information and thus censoring. Therefore, we extended our CNNs based on Diamant's study ${ }^{22}$ with the discrete-time survival model by Gensheimer et al. ${ }^{27}$. The same extension was also applied to the $\mathrm{CNN}+\mathrm{Clinical}$ and the $\mathrm{ANN}$. These networks were trained from scratch following the same workflow used for binary classification, additionally incorporating the time-to-event/follow-up times for every patient. The output of such networks is for every patient a DM-free probability curve, as shown in Fig. 2 for two selected patients with and without DM occurrence.

We first performed time-to-event analysis using the same image input as for the binary classification task, i.e. the CT masked with the primary and lymph node GTVs. Additionally, to analyse the importance of texture for the CNNs we performed an experiment: instead of giving the network the GTV with re-scaled Hounsfield Units inside (standard image input), we gave the networks a binary image, i.e. the GTV with all values inside set to +1 and everything outside the GTV set to -1 (see Supplementary Fig. S1). In this way we can exclude that during the learning process the networks are looking at anything else except from the tumor volume and 3D shape (for the $3 \mathrm{D}-\mathrm{CNN}$ ) or the tumor area and $2 \mathrm{D}$ shape (for the $2 \mathrm{D}-\mathrm{CNN}$ ). For the binary masking experiment 


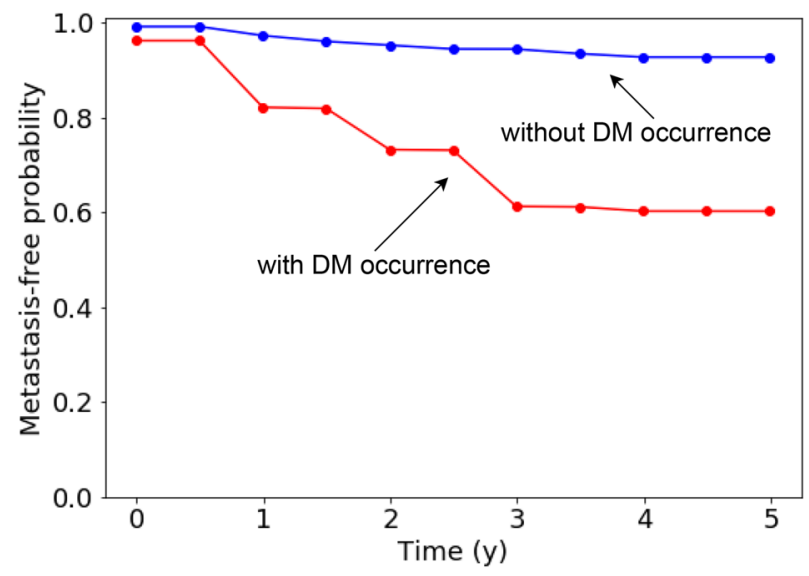

Figure 2. Time-to-event network output. The DM-free probability curves shown are taken from two exemplary patients of the $\mathrm{CRO}$ testing cohort using the $3 \mathrm{D}-\mathrm{CNN}$ as prognostic model. Note that the network output is at pre-defined discrete time points, the lines being drawn for visualisation purposes.

\begin{tabular}{|l|l|l|l|l|l|}
\hline Model & Image input & 3-fold CV & Test MAASTRO & Test PMH & Test CRO \\
\hline 2D-CNN & Standard & $0.74 ; 0.75 ; 0.71$ & $0.81(0.70-0.91)$ & $0.64(0.59-0.69)$ & $0.86(0.76-0.93)$ \\
\hline 2D-CNN+Clinical & Standard & $0.78 ; 0.88 ; 0.80$ & $0.88(0.82-0.94)$ & $0.67(0.61-0.71)$ & $0.77(0.66-0.87)$ \\
\hline 3D-CNN & Standard & $0.78 ; 0.74 ; 0.80$ & $0.88(0.80-0.93)$ & $0.67(0.62-0.71)$ & $0.77(0.60-0.90)$ \\
\hline 3D-CNN+Clinical & Standard & $0.82 ; 0.84 ; 0.88$ & $0.88(0.80-0.94)$ & $0.66(0.61-0.71)$ & $0.74(0.64-0.85)$ \\
\hline 2D-CNN & Binary & $0.67 ; 0.76 ; 0.64$ & $0.75(0.61-0.86)$ & $0.69(0.64-0.73)$ & $0.76(0.63-0.89)$ \\
\hline 2D-CNN+Clinical & Binary & $0.90 ; 0.74 ; 0.78$ & $0.87(0.81-0.93)$ & $0.66(0.61-0.70)$ & $0.74(0.64-0.83)$ \\
\hline 3D-CNN & Binary & $0.77 ; 0.78 ; 0.80$ & $0.87(0.80-0.92)$ & $0.67(0.62-0.72)$ & $0.77(0.65-0.87)$ \\
\hline 3D-CNN+Clinical & Binary & $0.90 ; 0.80 ; 0.77$ & $0.85(0.75-0.94)$ & $0.67(0.62-0.72)$ & $0.72(0.61-0.82)$ \\
\hline ANN & - & $0.78 ; 0.82 ; 0.76$ & $0.87(0.81-0.92)$ & $0.66(0.61-0.71)$ & $0.74(0.62-0.84)$ \\
\hline
\end{tabular}

Table 2. Comparison of HCIs for CV and testing for different time-to-event models and the two different image input scenarios. The numbers shown for the 3-fold CV are HCIs obtained for the CV subsets. Numbers for the testing sets represent the median $\mathrm{HCI}$ and in brackets the $83 \%$ confidence intervals obtained via bootstrap re-sampling. Note that HCIs for the ANN are shown only once because the only input are clinical variables of the corresponding patients.

we took the best hyper-parameters obtained with standard image input and then followed again the exact same data splitting and workflow as depicted in Fig. 1, to ensure that no information flows from the testing cohorts into the hyper-parameters used for training.

To evaluate the performance of time-to-event models on different cohorts we used $\mathrm{HCI}^{32}$ as it incorporates censoring in the computation of the metric and is therefore well suited to assess performance for this task.

Table 2 shows the results for $\mathrm{CV}$ and testing for both the standard image input and the binary masked image input. In general, trends from binary classification have been confirmed for the MAASTRO and the PMH cohort while we observed an overall improvement for the CRO cohort. Both the 2D/3D CNNs and the 2D/3D CNNs+Clinical were able to transfer good validation results to two out of three cohorts. The same holds for the ANN, although results were on average slightly worse than for the CNNs and the CNNs+Clinical. Again, the PMH dataset was found to be worst for all models.

Of interest is also the fact that when performing the binary masking experiment the HCI for the 3D-CNN and for the $3 \mathrm{D}-\mathrm{CNN}+\mathrm{Clinical}$ decreased by no more than 0.03 (i.e. not significantly). A more substantial drop (yet still not significant in terms of confidence intervals) was observed for the $2 \mathrm{D}-\mathrm{CNN}$ and the $2 \mathrm{D}-\mathrm{CNN}+\mathrm{Clinical}$.

In addition to the assessment of the discriminative power according to HCI, we also analysed patient stratification capability of the time-to-event networks. To do so, we found an optimal threshold to split the CV cohorts into high- and low-risk patients and then applied this threshold to the three independent testing cohorts. To infer whether the stratification on the testing cohorts was significant we applied the log rank test on the two patient groups. The resulting $\mathrm{p}$ value and corresponding Kaplan-Meier plots for the two groups are shown in Fig. 3 for the $3 \mathrm{D}-\mathrm{CNN}$ and the $2 \mathrm{D}-\mathrm{CNN}+\mathrm{Clinical}$ with standard image input (because the average performance over all testing cohorts was the best and equal for these two models) and for the ANN as baseline. A complete list containing the patient stratification $\mathrm{p}$ values for all tested models can be found as Supplementary Table S1.

As can be seen in Fig. 3a,b, the CNNs were able to significantly stratify all three testing cohorts into highand low-risk patient subgroups. Even though also the ANN significantly stratified all three testing cohorts (Fig. 3c), it should be noted that, as visible in the plots, the obtained difference between the two risk groups is 
a)
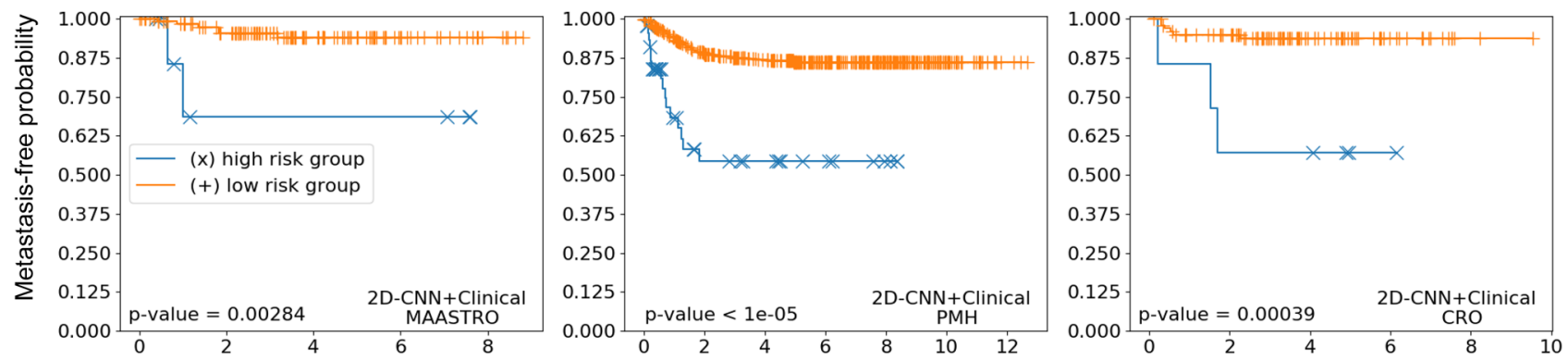

b)
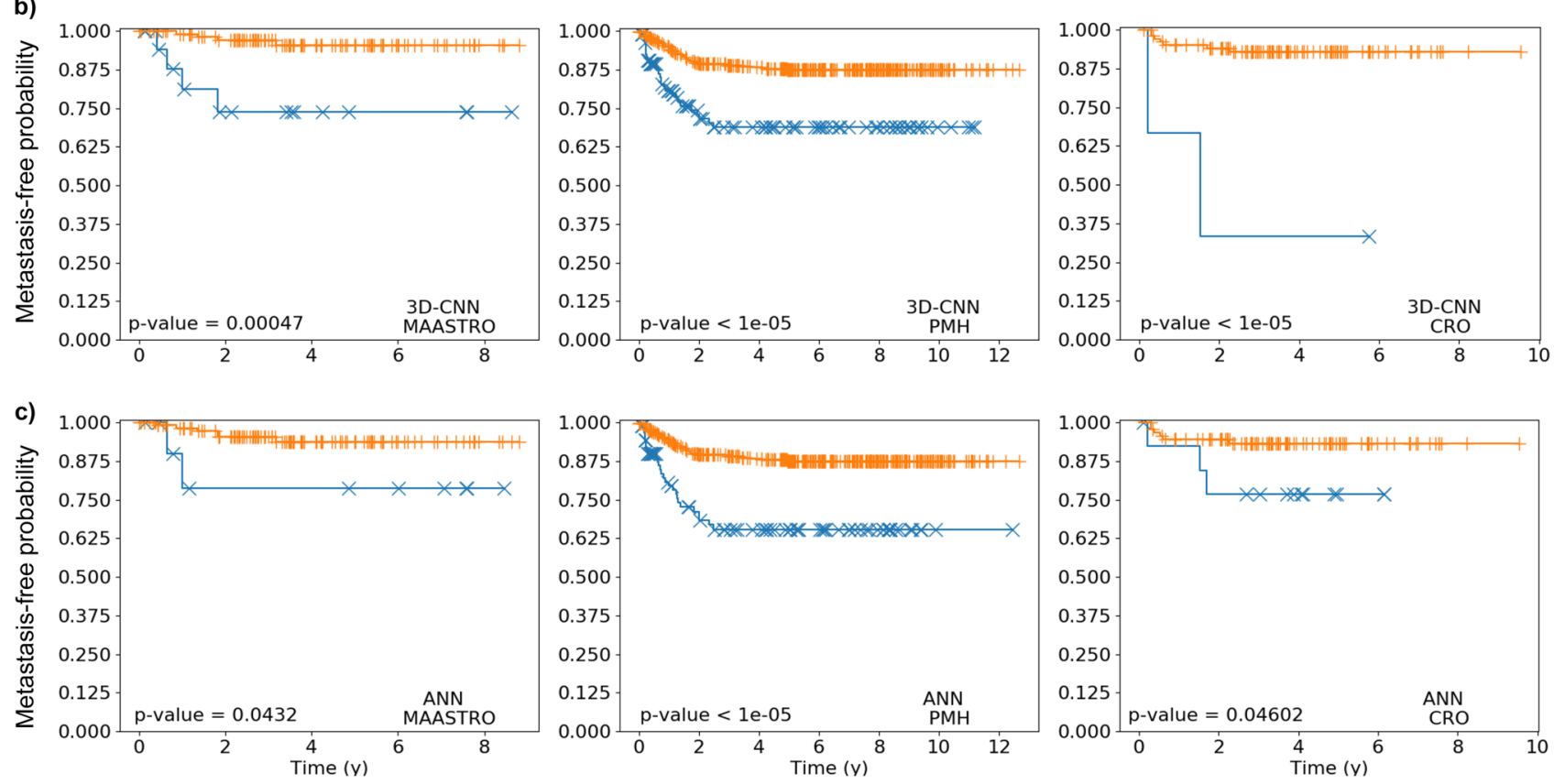

Figure 3. Kaplan-Meier curves obtained when applying time-to-event models on the 3 testing cohorts. The testing patients were stratified into two groups by using an optimised threshold obtained during CV. The displayed $\mathrm{p}$ values were computed using the log rank test. (a) Kaplan-Meier curves resulting from 2D-CNN+Clinical. (b) Kaplan-Meier curves resulting from 3D-CNN. (c) Kaplan-Meier curves resulting from ANN based on clinical variables.

less pronounced if compared to the $2 \mathrm{D} \mathrm{CNN}+$ Clinical and the 3D CNN in two (MAASTRO and CRO) out of three testing cohorts.

\section{Discussion}

In general, it was shown that $\mathrm{CT}$ based $2 \mathrm{D}$ and $3 \mathrm{D}$ CNNs are well performing and reliable models for DM time-to-event analysis in head and neck cancer patients. The binary classification $2 \mathrm{D}-\mathrm{CNN}$ built de novo in the benchmark study by Diamant et al. ${ }^{22}$ was chosen as a starting point, also making use of public data on the TCIA $^{23,24}$. Compared to Diamant et al. we used a 3D network in addition to a 2D network and both the primary and the lymph node GTVs instead of only the primary GTV. When assessing whether this increase in complexity was justified based on the CV cohort, we observed an average increase in performance when going from the 2D-CNN to the 3D-CNN (Table 1, 3rd column). This result suggested that some relevant information for DM outcome prediction is contained in the full $3 \mathrm{D}$ volume.

The benchmark study did not evaluate model performance on an independent test set. To infer whether results obtained on the validation set (on which the hyper-parameter tuning is performed) can be transferred to independent testing sets we gathered three additional data sets from three different institutions in North America (PMH) and Europe (MAASTRO, CRO) and applied our trained models to them (Fig. 1). As can be seen in Table 1, only the 2D-CNN was able to transfer good average CV AUCs to two out of three testing sets, contradicting the results obtained for $\mathrm{CV}$ alone and thus underlining the importance of using independent testing sets. To analyse whether a simpler model would be able to achieve similar results we also built an ANN based solely on clinical variables and obtained on average similar results to the $2 \mathrm{D}-\mathrm{CNN}$, although the values varied more between the different testing cohorts.

To see if combined clinical and image information leads to better performance we extended the $2 \mathrm{D}$ and 3D CNNs to include clinical covariates but obtained no improvement in performance when looking at testing 


\begin{tabular}{|l|l|l|l|l|l|}
\hline Cohort & Male/female & Median age (years) & Overall stage I/II/III/IV & Median GTVp + GTVn & DM \\
\hline Benchmark & $76 / 24 \%$ & 63 & $1 / 9 / 20 / 68 \%$ & $31.9 \mathrm{~cm}^{3}$ & $13.3 \%$ \\
\hline MAASTRO & $81 / 19 \%$ & 61 & $17 / 8 / 16 / 57 \%$ & $19.0 \mathrm{~cm}^{3}$ & $5.8 \%$ \\
\hline PMH & $80 / 20 \%$ & 61 & $1 / 6 / 14 / 78 \%$ & $39.4 \mathrm{~cm}^{3}$ & $14.3 \%$ \\
\hline CRO & $63 / 37 \%$ & 57 & $20 / 21 / 10 / 46 \%$ & $23.0 \mathrm{~cm}^{3}$ & $7.9 \%$ \\
\hline
\end{tabular}

Table 3. Comparison of clinical variables and percentage of patients having a DM among the different cohorts used in this study.

cohorts. It should be noted that we only used 7 clinical variables as these were the only ones available in all 4 cohorts. Using more covariates might increase the performance of the ANN and the CNN+Clinical.

To overcome the issue of patients loss during follow-up, we extended all architectures with the time-to-event model by Gensheimer et al. ${ }^{27}$. This allowed for incorporating censoring information in the training process and enabled output of DM-free probability curves at different time points for every patient (see Fig. 2). This could allow for better personalised treatments as the additional information on how fast an event occurs is available (which is particularly relevant for e.g. older patients). When looking at discriminative performance in terms of HCI we found that both the $2 \mathrm{D}$ and the $3 \mathrm{D}$ CNNs were able to transfer good validation results to two (MAASTRO and CRO) out of three testing cohorts (Table 2), unlike the binary classification CNNs. This suggests that incorporating censoring information may lead to a more consistent performance. The $2 \mathrm{D}-\mathrm{CNN}+\mathrm{Clinical}$ achieved exactly the same testing performance as the $3 \mathrm{D}-\mathrm{CNN}$ while the average testing $\mathrm{HCI}$ of the $2 \mathrm{D}-\mathrm{CNN}$, the $3 \mathrm{D}-\mathrm{CNN}+\mathrm{Clinical}$ and the ANN was slightly worse.

Although results were significantly better than 0.5 , the $\mathrm{PMH}$ cohort was the worst for both tasks, leading at best to an AUC of 0.66 (83\% CI 0.61-0.71) and a HCI of 0.69 (83\% CI 0.64-0.73). Considering the substantial GTV volume difference between the other cohorts and PMH (see Table 3) we exploited the high number of patients in this cohort (497) to tackle the performance problem by training time-to-event CNNs and ANNs from scratch using only the PMH cohort. The best hyper-parameters were found by applying 3-fold CV several times on the first $50 \%$ of the cohort. Again, the three CV models leading to the best validation HCI were then applied on the second $50 \%$ of the cohort (using model averaging and bootstrap re-sampling). However, we achieved no improvement on average, the best result being a HCI of 0.69 (see Supplementary Table S2). In a previously published study, Kwan et al. ${ }^{4}$ had also used a subset of $\mathrm{PMH}$ with a traditional radiomics model to discriminate DM risk. Their best HCI of 0.71 is in good agreement with our results, hinting towards the fact that no matter which models are used, some cohorts might be more challenging than others, at least in terms of discriminative power.

In this work, we also performed a binary masking experiment on the $\mathrm{CNN}$ input image. Instead of giving the network the GTV contours with re-scaled Hounsfield Units inside, we set all values inside the GTV to +1 and then fed the resulting image to the time-to-event CNNs (see Supplementary Fig. S1). The performance of the networks did not change significantly for both the $2 \mathrm{D}-\mathrm{CNN}$ and the $3 \mathrm{D}-\mathrm{CNN}$, although a more notable drop was observed for the 2D-CNN (Table 2). These results suggest that while tumor texture might increase performance if the CNN is limited to 2D, it is of less relevance for the 3D-CNN, the volume and 3D shape of the GTVp and GTVn being sufficient to achieve good testing performances. The latter finding is in agreement with the result obtained by Welch et al. ${ }^{9}$ using a traditional radiomics model.

Finally, we also assessed the performance of our models in terms patient stratification capability. To prevent information flow from testing to training/validation we found a threshold to split patients in high- and lowrisk groups using the validation cohort and then applied this threshold to the three testing cohorts. Our best performing models in terms of $\mathrm{HCI}$, that is, the $2 \mathrm{D}-\mathrm{CNN}+\mathrm{Clinical}$ and the $3 \mathrm{D}-\mathrm{CNN}$ with standard image as input, were also found to achieve good results for patient stratification, being able to significantly separate all three testing cohorts (log-rank test p values $<0.05$ ). Clinically speaking, this result might be of relevance as CNNs could be used to determine risk groups that might not respond well, or respond better. This information could then be used for treatment (or dose level) adaptation. The ANN was slightly worse than the CNNs, the separation between the high-risk and the low-risk groups being less pronounced for the MAASTRO and CRO cohorts, as can be seen in Fig. 3.

Recently, a study with 291 patients investigating different deep learning approaches for predicting locoregional head and neck tumor control was published ${ }^{33}$. Censoring information was incorporated in all models using a different time-to-event model than in our study ${ }^{26}$. Similarly to this work, the authors found a CT based time-to-event 3D-CNN to be the best performing model, both in terms of discriminative power as measured with $\mathrm{HCI}$ and in terms of patient stratification capability. Regarding the performance of their baseline clinical model in terms of HCI, a more considerable drop with respect to the 3D-CNN was found. In contrast to our study, they found the 2D-CNNs to be substantially worse than the 3D-CNNs, although it should be kept in mind that the prognosis endpoint was a different one.

The main drawback of this study consists in its retrospective nature. Consequently, not all clinical background information was available. For example, for many patients there is uncertainty on whether they received surgery after the primary radiotherapy treatment (see "Patient cohorts" subsection), a fact which might change the outcome of the treatment. For the PMH cohort this information is missing for all patients which might be one of the reasons for its underperformance. Therefore, large prospective studies could be important in the assessment of outcome prediction models. Additionally, as a consequence of our findings it is even more crucial to reduce interphysician variability in the contouring stage, as the binary masking experiment underlined the importance of 
tumor volume and shape. Future studies could address whether more advanced CNN architectures like ResNet ${ }^{34}$ or DenseNet ${ }^{35}$ improve DM outcome prediction. It also remains to be seen if additional imaging (e.g. positron emission tomography or magnetic resonance imaging) or genomic biomarkers can enhance the performance of deep learning algorithms for binary classification or time-to-event prediction.

In conclusion, this report highlighted via a large number of independent testing patients the efficacy of image based deep learning models for DM binary classification and time-to-event analysis.

\section{Methods}

Patient cohorts. For this study, 4 different cohorts totalling 1037 patients with head and neck cancer were used. All patients selected for this study received either radiation alone or chemo-radiation as main treatment and have a follow-up time larger than 2 years. All patients which had a metastasis at time of diagnosis were excluded.

- Canadian benchmark cohort: consists of 298 patients treated at 4 different hospitals in Quebec, Canada. Out of 298 patients, 294 were used for this study as few patients had to be excluded due to data corruption or non-clear identification of the RTSTRUCT corresponding to the CT. This cohort was used in the benchmark study by Diamant et $\mathrm{al}^{22}$ and in a traditional radiomics study ${ }^{5}$ on which the former was based. All patients received either radiation alone or chemo-radiation as main treatment modality. For three out of four hospitals information on surgery was not available. Out of the 88 patients for whom surgery information was available, 10 received it. More detailed information on this cohort can be found on TCIA ${ }^{23}$.

- MAASTRO cohort: consists of 137 patients treated at Maastricht Radiation Oncology clinic in the Netherlands. Out of 137 patients, 1 had to be excluded due to metastasis at first diagnosis. This cohort was used for survival analysis by Aerts et al. $^{3}$ in one of the first traditional radiomics studies, but to our knowledge it was never used to predict DM outcome. All patients were treated with radiotherapy and for all patients information on surgery was available: on 6 out of 136 cancer surgery was performed. More information on this cohort can be found on TCIA ${ }^{30}$.

- PMH cohort: consists of 606 patients treated at Princess Margaret Cancer Centre in Canada. Out of 606, 497 were used for this study as the remaining ones had to be exluded due to short follow-up time, missing RTSTRUCT or missing GTV for instance due to surgical resection. Although information on surgery was not available in the clinical data sheet, contour names for some patients suggested that a surgery or a resection was performed. All patients were treated with either radiotherapy alone or chemo-radiotherapy as primary treatment. Subsets of this cohort were used in several studies ${ }^{4,9,36}$, although only Kwan et al. applied a traditional radiomics model on it to discriminate DM risk. More detailed information on this cohort can be found on TCIA ${ }^{31}$.

- CRO cohort: consist of 110 patients treated at Centro di Riferimento Oncologico Aviano in Italy. Only those patients were selected who were concordant with the other cohorts in terms of clinical specifications (e.g. tumor site, treatment modality, etc.). All patients were treated with either radiation alone or with chemoradiation as primary treatment and no patient received surgery. This cohort was obtained via a collaboration and can be shared upon request.

For all patients, the pre-treatment CT and primary (GTVp) and lymph node (GTVn) gross tumor volumes contoured by expert physicians of the corresponding hospitals were available. For all cohorts but PMH the GTVns were clearly labeled in the RTSTRUCT. For PMH every region of interest was contoured, thus to select the GTVn we looked into every patient manually and labeled as GTVns those nodes which were inside the clinical target volume. After that, under consultation of an expert physician in our department, we excluded all previously selected lymph nodes which had a volume smaller than $2 \mathrm{~cm}^{3}$. Table 3 compares some relevant clinical variables and specifics among the different cohorts used in this study.

The Canadian benchmark cohort, MAASTRO and PMH are publicly available datasets and were retrieved from TCIA in anonymised form. The CRO patient data was analysed retrospectively, in anonymised form and are part of two studies approved by the Unique Regional Ethics Committee, with following approval numbers: CRO-2017-50 and CRO-2019-66. All methods were carried out in accordance with relevant guidelines and regulations. Informed consent was obtained from all patients.

Network architectures. Both our 2D-CNN and our 3D-CNN architectures are inspired by the 2D-CNN built by Diamant et al. ${ }^{22}$. As depicted in Fig. 4, our 3D-CNN (and 2D-CNN) comprises 3 convolutional blocks followed by two fully connected layers, one dropout layer and the final output layer. Every convolutional block is formed by a convolutional layer, which allows to change the representation of the input data while taking into account spatial information, a max-pooling layer, which introduces invariance of the network to small translations of the input ${ }^{11}$ and drastically reduces the number of parameters, and a parametric rectified linear unit (PReLu) as non-linear activation function (not shown in Fig. 4). After the convolutional blocks, the input image has been translated into a set of features with shape $(128,1,1,1)$ which are flattened to a vector of shape $(128$, which in turn is given as input to the two following fully connected layers (again followed by a PReLu activation function which is not shown in Fig. 4). After that follows a dropout layer, in which a number of neurons given by the dropout_rate hyper-parameter is set to zero to prevent the network from over-fitting, and finally the last fully connected layer which, together with a sigmoid activation function (not shown), forms the output of the network For the binary classification network this is a single number between 0 and +1 . 


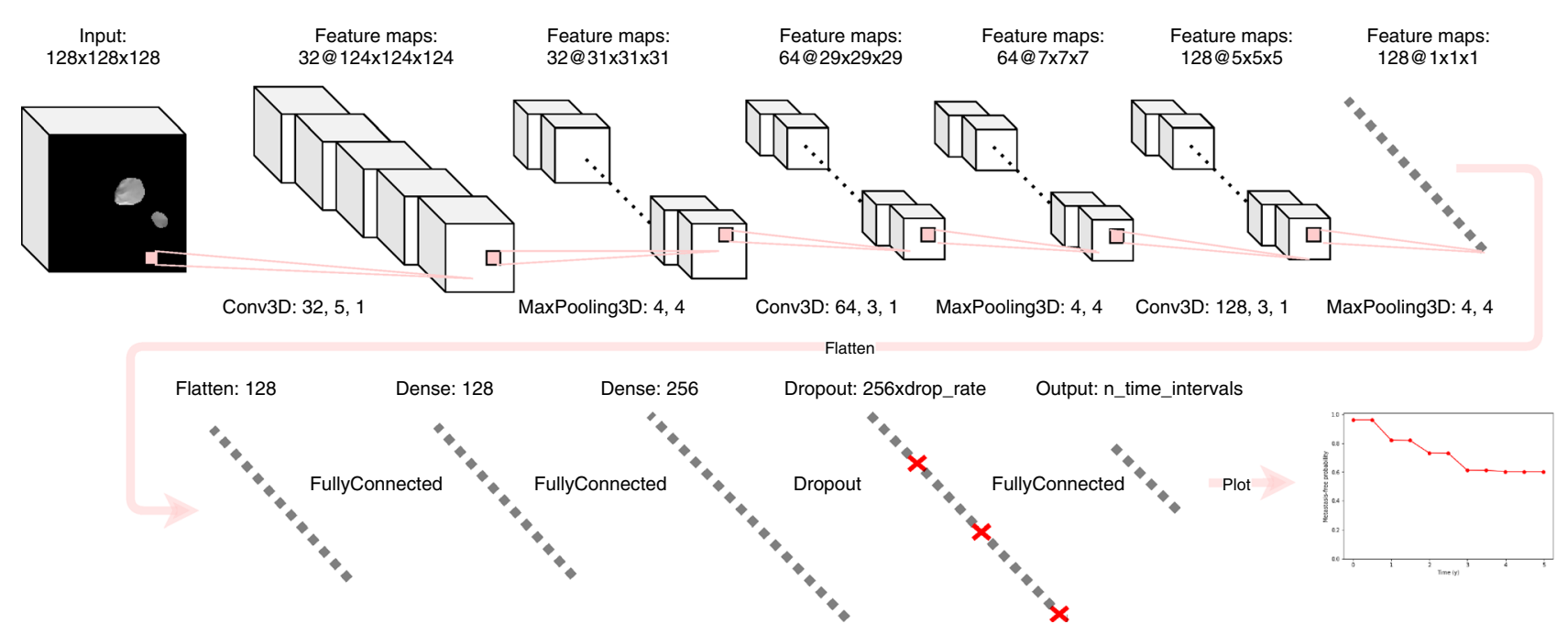

Figure 4. Time-to-event $3 \mathrm{D}$ convolutional neural network architecture. The general network architecture is based on the $2 \mathrm{D} \mathrm{CNN}$ implemented by Diamant et al. ${ }^{22}$ plus the discrete time-to-event model by Gensheimer et al. ${ }^{27}$. Numbers for the convolutional layers represent the number of kernels, the kernel size and the stride. Numbers for the max-pooling layers represent the pool size and the stride.

The main differences of our network with respect to the benchmark study ${ }^{22}$ are that we used both a 3D-CNN and a $2 \mathrm{D}-\mathrm{CNN}$ and that we extended the CNNs with the survival model by Gensheimer et al. ${ }^{27}$ to be able to incorporate time-to-event information.

To extend the network from $2 \mathrm{D}$ to $3 \mathrm{D}$ we used $3 \mathrm{D}$ Keras built-in functions for the convolutions and the max-pooling instead of the corresponding $2 \mathrm{D}$ functions. The image input for our $2 \mathrm{D}$-CNN was the $256 \times 256$ axial slice with the highest number of tumor pixels as in Diamant's study ${ }^{22}$. The input for the 3D-CNN was the full 3D image of $256 \times 256 \times 256$. To avoid memory issues both the $2 \mathrm{D}$ and the $3 \mathrm{D}$ input image were reduced to $128 \times 128(\times 128)$ with the random cropping augmentation. To adapt the binary classification CNNs to time-toevent analysis the only change which had to be done at architecture level was to increase the number of neurons in the final layer from one to $n \_$time_intervals, i.e. to the number of time intervals for which the network outputs an event probability (see Fig. 4). More details on the implementation of the discrete-time survival model ${ }^{27}$ within our framework can be found in the next subsection.

To compare the performance of the $\mathrm{CNN}$ with a simple baseline model based solely on clinical variables we opted for a shallow artificial neural network. To be specific, our ANN has seven clinical variables as input for every patient, that is, patient's age, gender, tumor site, overall stage, T-stage, $\mathrm{N}$-stage and primary plus lymph node tumor volume. Age in years and total tumor volume in $\mathrm{cm}^{3}$ were divided by 100 and given directly to the ANN while the other five variables, being of categorical nature, were one-hot encoded and then given to the ANN. The input layer of the network was followed by one fully connected hidden layer with 14 neurons, a ReLu activation function, a dropout layer and the final fully connected layer with sigmoid activation function. Exactly as for the $\mathrm{CNN}$, the last layer of the $\mathrm{ANN}$ has 1 neuron for the binary classification task and n_time_intervals neurons for the time-to-event task.

Finally, in an attempt to get a better performance by combining the two previous models, we also constructed what we call the $\mathrm{CNN}+\mathrm{Clinical}$. This network has the same architecture as the CNN but has as an additional input to the masked CT also the same seven clinical variables which were used for the ANN. The clinical variables are given to the $\mathrm{CNN}+\mathrm{Clinical}$ at the level of the flattening layer by concatenating the 128 features from the convolutional blocks with the vector containing the seven (partly) one-hot encoded clinical covariates. The resulting vector is then fed to the remaining architecture with fully connected layers exactly as for the normal CNN (Fig. 4).

Time-to-event analysis. Although binary classification models as the ones built in ${ }^{5,17,22}$ achieve good prediction results, they are limited by the fact that time-to-event information is discarded during model training. Especially in some situations, for instance when predicting death or metastasis occurrence for older patients, it is fundamental for treatment customisation to know how fast the event would occur. Additionally, binary classification models usually are trained on a specific time point (e.g. 2-year OS) so they would need to be re-trained to make predictions for a different one ${ }^{27}$. To overcome these issues we extended Diamant's network to include timeto-event information by incorporating the survival model by Gensheimer et al. ${ }^{27}$. Their discrete-time survival model has been implemented in Keras ${ }^{37}$ (https://github.com/MGensheimer/nnet-survival) and allows to train deep neural networks while taking into account patient follow-up times and outputs discrete event probability/ survival curves for each patient. To our knowledge, the only applications of Gensheimer's model to the field of medical imaging are the study by Zhang et al. ${ }^{38}$, who recently showed that their CT-based CNN survival model was able to outperform a Cox proportional hazards model based on radiomic features for pancreatic ductal adenocarcinoma and the study by Kim et al. ${ }^{39}$, who developed a CT-based deep learning model which successfully predicts disease-free survival for lung adenocarcinoma patients. 
To train our time-to-event CNN, the custom loss function surv_likelihood provided by the authors is used. At architecture level, the number of neurons in the final connected layer has to be changed in order to match the desired number of output time intervals (Fig. 4). To be specific, every neuron in the final layer outputs the conditional probability that the patient does not get a DM in the corresponding time interval. To obtain a "survival curve" as displayed in Fig. 4 the conditional probabilities up to a certain time interval have to be cumulatively multiplied. For our experiments we used 10 time intervals with a spacing of half a year.

To evaluate the performance by taking into account time-to-event information (conversely to the AUC) we used $\mathrm{HCI}^{32}$ which is defined as the fraction of patients for which the predictions and the outcomes are concordant. For our purposes we adapted Lifelines' concordance index (https://lifelines.readthedocs.io/en/latest/ lifelines.utils.html\#lifelines.utils.concordance_index) function to compute the concordance between a patient's metastasis-free probability after 3 years and the patient's ground truth event time. Following this definition, a perfect HCI for our model would be 1.0, as patients with e.g. a low value for metastasis-free probability should be patients with a short time-to-event.

Implementation details. All the code needed for data pre-processing and running the models was written in Python 3.6. The networks were built and optimized with Tensorflow 2.2.0 ${ }^{40}$ using the high-level library $\mathrm{Keras}^{37}$. Training and testing was carried out on three different graphical processing units: a NVIDIA P6000 with 24 GB, a NVIDIA P5000 with 16 GB and a NVIDIA Titan V with 12 GB of memory. The input shape of the 3D CT of $256 \times 256 \times 256$ and the batchsize of four were chosen so that the networks could fit also on the smallest graphical processing unit available. For the $2 \mathrm{D}-\mathrm{CNNs}$ a batchsize of 32 was used. The weights and biases of the networks were optimized using the Adam algorithm ${ }^{41}$ with a constant learning rate of $3 \times 10^{-4}$ and $2 \times 10^{-4}$ for the 2D and 3D binary classification CNNs and of $6 \times 10^{-4}$ and $3 \times 10^{-4}$ for the $2 \mathrm{D}$ and 3D time-to-event CNNs. The respective loss functions used were binary cross-entropy and the custom loss function surv_likelihood by Gensheimer et al. ${ }^{27}$.

Prior to the augmentations, all images were isotropically re-sampled to a $1 \times 1 \times 1 \mathrm{~mm}^{3}$ grid. Specifically, the binary masks where re-sampled in 3D using in-house code for shape-based interpolation ${ }^{42}$. The full CTs where interpolated in $3 \mathrm{D}$ using linear interpolation. Moreover, all CT values were windowed to -500 and +500 Hounsfield Units and then re-scaled from -1 to +1 (standard image input). The linearly interpolated CTs were then masked with the shape-based interpolated binary masks to create the re-sampled masked CTs which were used as input to the networks. For the binary masking experiment, we set all values inside the GTV which were bigger than -1 to +1 , i.e. we used re-sampled binary masks as input (see Supplementary Fig. S1).

To decrease over-fitting, a weight decay term of $1 \times 10^{-4}$ was used for all models. Additionally, for the $2 \mathrm{D}$ and $3 \mathrm{D}$ CNNs and the $2 \mathrm{D}$ and $3 \mathrm{D} \mathrm{CNNs}+$ Clinical several augmentations were implemented by adapting a multithreaded augmentation pipeline which is freely available online ${ }^{43}$ (https://github.com/MIC-DKFZ/batch generators) to our workflow. This allowed us to use a slightly wider range of augmentations and especially to perform the augmentations online (during the training), in contrast to Diamant's study. To be specific, we used random cropping to 128 pixels in each dimension with a central random shift of maximal $20 \%$ of the original $256 \times 256 \times 256$ image. We also used mirroring of the image with $50 \%$ probability, rotations of maximal 60 degrees and elastic deformations of the image of up to $25 \%$ of the size of the cropped image, both with a probability of $10 \%$ for the binary classification $3 \mathrm{D}-\mathrm{CNN}, 20 \%$ for the time-to-event $3 \mathrm{D}-\mathrm{CNN}$ and $50 \%$ for all 2D-CNNs. Finally, for all our models we also used dropout with a dropout_rate of $25 \%$. For the CNNs all these hyper-parameters were found by performing a manual grid search meaning that we repeated the 3-fold CV on the Canadian benchmark data set until we were satisfied with the mean validation AUC or HCI which was achieved with given parameters. On the other hand, training for the ANN took much less, so after manually finding a range of parameters leading to good results we performed an automatic grid search over 16 different combinations of hyper-parameters (number of neurons in the hidden layer, weight decay, dropout probability, learning rate) to find the ones performing best on average for the 3-fold CV. For all CNN+Clinical models we manually explored some hyper-parameters around the best hyper-parameters for the CNNs and found that the ones leading to best average CV were the same as for the CNN.

For all our network architectures we took the 3 models achieving the best average CV performance and used them for testing without any further change. To get a single prediction out of three we applied the validation models on test data and then averaged the resulting three predictions to obtain a single model averaged prediction per patient. A schematic view of the workflow is shown in Fig. 1.

When doing binary classification the 3D-CNN and 3D-CNN+Clinical were trained for 500 epochs, using Keras callbacks to save the model's weights when an improvement in the AUC was observed. One 3-fold CV took on average $11 \mathrm{~h}$. The $2 \mathrm{D}-\mathrm{CNN}$ and $2 \mathrm{D}-\mathrm{CNN}+\mathrm{Clinical}$ were trained for 500 epochs, one 3 -fold CV taking on average 30 minutes. The ANN was trained for 5000 epochs, one 3 -fold CV taking on average 10 minutes. When performing time-to-event analysis the 2D and 3D CNNs and CNNs+Clinical were trained for 300 epochs while the ANN for 3000, taking on average $8 \mathrm{~h}$ for the 3D-CNNs, $20 \mathrm{~min}$ for the $2 \mathrm{D}-\mathrm{CNNs}$ and around $10 \mathrm{~min}$ for the ANN. For all the networks and the tasks, we used early stopping with a patience of 200 for all CNNs and 2000 for the ANN to shorten the overall training time.

Statistical analysis. The performance of the different models on the different testing cohorts was investigated in a two-fold way: by looking at the discriminative power using the area under the receiver operating characteristic curve $^{44}$ (AUC) for binary classification tasks or Harrell's concordance index ${ }^{32}$ (HCI) for time-to-event tasks and by looking at patient stratification capability using a threshold optimised on the validation cohort. To find the threshold we first averaged over the risk of all validation patients who got a DM, then we averaged over the risk of all the validation patients which did not get a DM and finally we took the mean of these two 
thresholds for all three CV models and obtained a final model averaged threshold to be used for stratification of the testing sets.

Following the suggestions by ${ }^{45,46}$ we assessed significance of difference by using both estimation statistics and statistical tests. We applied estimation statistics ${ }^{45}$, i.e. we focused on the sizes of effects at level of data, by computing the median AUC or HCI with $83 \%$ confidence intervals from bootstrap re-sampling (consisting in generating many variants of a given dataset by repeatedly taking samples with replacement from the original set $^{32}$ ). We used $83 \%$ confidence as it can be shown ${ }^{47,48}$ that two non-overlapping $83 \%$ confidence intervals mean that the two corresponding means/medians differ significantly with a significance level of 0.05 . To be in line with literature ${ }^{5,9,17}$, we also split the testing patients into a high- and low-risk group and checked whether the stratification is significant by computing the $\mathrm{p}$ value for the log-rank test. We consider results with $\mathrm{p}$ values $<0.05$ to be statistically significant.

\section{Code availability}

All the code needed to build the models and perform the analysis is freely available on GitLab: https:/gitlab. physik.uni-muenchen.de/LDAP_ag-E2ERadiomics/dl_based_prognosis.

\section{Data availability}

Three out of four cohorts used in this study can be found on The Cancer Imaging Archive (TCIA) ${ }^{24}$ : Canadian

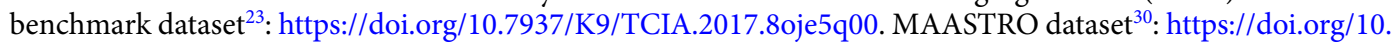
7937/tcia.2019.8kap372n. PMH dataset ${ }^{31}$ : https://doi.org/10.7937/tcia.2019.8dho2gls. The CRO dataset is the result of a collaboration and can be obtained upon request.

Received: 17 September 2020; Accepted: 28 February 2021

Published online: 19 March 2021

\section{References}

1. Baumann, M. et al. Radiation oncology in the era of precision medicine. Nat. Rev. Cancer 16(4), 234-249. https://doi.org/10.1038/ nrc.2016.18 (2016).

2. Morin, O. et al. A deep look into the future of quantitative imaging in oncology: A statement of working principles and proposal for change. Int. J. Radiat. Oncol. Biol. Phys. 102(4), 1074-1082. https://doi.org/10.1016/j.ijrobp.2018.08.032 (2018).

3. Aerts, H. et al. Decoding tumour phenotype by noninvasive imaging using a quantitative radiomics approach. Nat. Commun. $\mathbf{5}$, 4006. https://doi.org/10.1038/ncomms5006 (2014).

4. Kwan, J. Y. Y. et al. Radiomic biomarkers to refine risk models for distant metastasis in HPV-related oropharyngeal carcinoma. Int. J. Radiat. Oncol. Biol. Phys. 102(4), 1107-1116. https://doi.org/10.1016/j.ijrobp.2018.01.057 (2018).

5. Vallieres, M. et al. Radiomics strategies for risk assessment of tumour failure in head-and-neck cancer. Sci. Rep. 7, 1-33. https:// doi.org/10.1038/s41598-017-10371-5 (2017).

6. Buizza, G. et al. Early tumor response prediction for lung cancer patients using novel longitudinal pattern features from sequential pet/ct image scans. Phys. Med. 54, 21-29. https://doi.org/10.1016/j.ejmp.2018.09.003 (2018).

7. Gilies, R. J. et al. Radiomics: Images are more than pictures, they are data. Radiology 278(2), 563-577. https://doi.org/10.1148/ radiol.2015151169(2016).

8. Avanzo, M., Stancanello, J. \& El Naqa, I. Beyond imaging: The promise of radiomics. Phys. Med. 38, 122-139. https://doi.org/10. 1016/j.ejmp.2017.05.071 (2017).

9. Welch, M. L. et al. Vulnerabilities of radiomic signature development: The need for safeguards. Radiother. Oncol. 130, 2-9. https:// doi.org/10.1016/j.radonc.2018.10.027 (2019).

10. Avanzo, M. et al. Machine and deep learning methods for radiomics. Med. Phys. 47(5), e185-e202. https://doi.org/10.1002/mp. 13678 (2020).

11. Goodfellow, I., Bengio, Y. \& Courville, A. Deep Learning (MIT Press, 2016).

12. Shen, C. et al. An introduction to deep learning in medical physics: Advantages, potential, and challenges. Phys. Med. Biol.https:// doi.org/10.1088/1361-6560/ab6f51 (2020).

13. LeCun, Y., Bengio, Y. \& Hinton, G. Deep learning. Nature 521(7553), 436-44. https://doi.org/10.1038/nature14539 (2015).

14. Esteva, A. et al. Dermatologist-level classification of skin cancer with deep neural networks. Nature 542, 115-118. https://doi.org/ 10.1038 /nature21056 (2017).

15. Kann, B. H. et al. Multi-institutional validation of deep learning for pretreatment identification of extranodal extension in head and neck squamous cell carcinoma. J. Clin. Oncol. 38(12), 1304-1311. https://doi.org/10.1200/JCO.19.02031 (2020).

16. Trebeschi, S. et al. Deep learning for fully-automated localization and segmentation of rectal cancer on multiparametric mr. Sci. Rep. 7, 5301. https://doi.org/10.1038/s41598-017-05728-9 (2017).

17. Hosny, A. et al. Deep learning for lung cancer prognostication: A retrospective multi-cohort radiomics study. PLoS Med. 15(11), e1002711. https://doi.org/10.1371/journal.pmed.1002711 (2018).

18. Kooi, T. et al. Large scale deep learning for computer aided detection of mammographic lesions. Med. Image Anal. 35, $303-312$. https://doi.org/10.1016/j.media.2016.07.007 (2017).

19. Hansen, D. C. et al. Scatternet: A convolutional neural network for cone-beam CT intensity correction. Med. Phys. 46(5), 2538. https://doi.org/10.1002/mp.13175 (2019).

20. Han, X. MR-based synthetic CT generation using a deep convolutional neural network method. Med. Phys.https://doi.org/10. 1002/mp.12155 (2017).

21. Spadea, M. F. et al. Deep convolution neural network (DCNN) multiplane approach to synthetic CT generation from MR imagesapplication in brain proton therapy. Int. J. Radiat. Oncol. Biol. Phys. 105(3), 495-503. https://doi.org/10.1016/j.ijrobp.2019.06.2535 (2019).

22. Diamant, A., Chatterjee, A., Vallières, M., Shenouda, G. \& Seuntjens, J. Deep learning in head and neck cancer outcome prediction. Sci. Rep. 9(1), 2764. https://doi.org/10.1038/s41598-019-39206-1 (2019).

23. Vallieres, M. et al. Data from head-neck-PET-CT. Cancer Imaging Arch.https://doi.org/10.7937/K9/TCIA.2017.8oje5q00 (2017).

24. Clark, K. et al. The cancer imaging archive (TCIA): Maintaining and operating a public information repository. J. Digit. Imaging 26(6), 1045-1057. https://doi.org/10.1007/s10278-013-9622-7 (2013).

25. Ching, T., Zhu, X. \& Garmire, L. X. Cox-nnet: An artificial neural network method for prognosis prediction of high-throughput omics data. PLoS Comput. Biol. 14(4), e10006076. https://doi.org/10.1371/journal.pcbi.1006076 (2018).

26. Katzman, J. L. et al. Deepsurv: Personalized treatment recommender system using a cox proportional hazards deep neural network. BMC Med. Res. Methodol. 18(1), 24. https://doi.org/10.1186/s12874-018-0482-1 (2018). 
27. Gensheimer, M. F. \& Narasimhan, B. A scalable discrete-time survival model for neural networks. PeerJ 7, e6257. https://doi.org/ $10.7717 /$ peerj.6257 (2019).

28. Bray, F. et al. Global cancer statistics 2018: GLOBOCAN estimates of incidence and mortality worldwide for 36 cancers in 185 countries. CA Cancer J. Clin. 68(6), 394-424. https://doi.org/10.3322/caac.21492 (2018).

29. Cramer, J. D. et al. The changing therapeutic landscape of head and neck cancer. Nat. Rev. Clin. Oncol. 16, 669-683. https://doi. org/10.1038/s41571-019-0227-z (2019).

30. Wee, L. \& Dekker, A. Data from head-neck-radiomics-HN1. Cancer Imaging Arch.https://doi.org/10.7937/tcia.2019.8kap372n (2019).

31. Kwan, J. Y. Y. et al. Data from radiomic biomarkers to refine risk models for distant metastasis in oropharyngeal carcinoma. Cancer Imaging Arch.https://doi.org/10.7937/tcia.2019.8dho2gls (2019).

32. Harrell, F. E. J., Lee, K. L. \& Mark, D. Multivariable prognostic models: Issues in developing models, evaluating assumptions and adequacy, and measuring and reducing errors. Stat. Med. 15(4), 361-387. https://doi.org/10.1002/(SICI)1097-0258(19960229)15: 4<361::AID-SIM168>3.0.CO;2-4 (1996).

33. Starke, S. et al. 2D and 3D convolutional neural networks for outcome modelling of locally advanced head and neck squamous cell carcinoma. Sci. Rep. 10, 15625. https://doi.org/10.1038/s41598-020-70542-9 (2020).

34. He, K., Zhang, X., Ren, S. \& Sun, J. Deep residual learning for image recognition. In 2016 IEEE Conference on Computer Vision and Pattern Recognition (CVPR) 770-778. https://doi.org/10.1109/CVPR.2016.90 (2016).

35. Huang, G., Liu, Z., Van Der Maaten, L. \& Weinberger, K. Q. Densely connected convolutional networks. 2017 IEEE Conference on Computer Vision and Pattern Recognition (CVPR) 2261-2269. https://doi.org/10.1109/CVPR.2017.243 (2017).

36. Leijenaar, R. T. H. et al. External validation of a prognostic CT-based radiomic signature in oropharyngeal squamous cell carcinoma. Acta Oncol. 54(9), 1423-1429. https://doi.org/10.3109/0284186X.2015.1061214 (2015).

37. Chollet, F. keras. https://github.com/fchollet/keras (2015).

38. Zhang, Y. et al. CNN-based survival model for pancreatic ductal adenocarcinoma in medical imaging. BMC Med. Imaging $20,11$. https://doi.org/10.1186/s12880-020-0418-1 (2020).

39. Kim, H., Goo, J. M., Lee, K. H., Kim, Y. T. \& Park, C. M. Preoperative CT-based deep learning model for predicting disease-free survival in patients with lung adenocarcinomas. Radiology 00, 1-9. https://doi.org/10.1148/radiol.2020192764 (2020).

40. Abadi, M. et al. Tensorflow: Large-scale machine learning on heterogeneous distributed systems. In 12th USENIX Symposium on Operating Systems Design and Implementation (2016).

41. Kingma, D. \& Ba, J. Adam: A method for stochastic optimization. In International Conference on Learning Representations (2014).

42. Herman, G. T., Zheng, J. \& Bucholtz, C. A. Shape-based interpolation. IEEE Comput. Graph. Appl. 12, 69-79. https://doi.org/10. $1109 / 38.135915(1992)$

43. Isensee, F. et al. batchgenerators - a python framework for data augmentation. https://doi.org/10.5281/zenodo.3632567.

44. Bradley, A. P. The use of the area under the ROC curve in the evaluation of machine learning algorithms. Pattern Recogn. 30(7), 175-177. https://doi.org/10.1016/S0031-3203(96)00142-2 (1997).

45. Claridge-Chang, A. \& Assam, P. N. Estimation statistics should replace significance testing. Nat. Methods 13(2), 108-109. https:// doi.org/10.1038/nmeth.3729 (2016).

46. Du-Prel, J., Hommel, G., Roehrig, B. \& Blettner, M. Confidence interval or p value? Part 4 of a series on evaluation of scientific publications. Dtsch. Arztebl. Int. 106(19), 335-9. https://doi.org/10.3238/arztebl.2009.0335 (2009).

47. Austin, P. C. \& Hux, J. E. A brief note on overlapping confidence intervals. J. Vasc. Surg. 36, 194-5. https://doi.org/10.1067/mva. 2002.125015 (2002).

48. Goldstein, H. \& Healy, M. J. R. The graphical presentation of a collection of means. J. R. Stat. Soc. Ser. A 158(1), 175-177. https:// doi.org/10.2307/2983411 (1995).

\section{Acknowledgements}

We would like to thank Philipp Wesp, Balthasar Schachtner and Micheal Ingrisch from the Department of Radiology of the University Hospital of the LMU Munich for the help in setting up the MIC-DKFZ augmentation pipeline. Special thanks go to Leonard Wee and Andre Dekker from MAASTRO, and to Scott Bratman from $\mathrm{PMH}$ for answering our inquiries about the datasets. Elia Lombardo acknowledges Moritz Rabe for the support for the figures shown in this work and Sophie Duque for the precious help and contribution to the shape-based interpolation code.

\section{Author contributions}

G.L. and M.R. conceived the project. G.L., M.R., C.K. and E.L. pre-processed the data, analysed the results and wrote the manuscript. E.L. built the networks and analysed the data. M.A., V.G., G.F., G.F. and J.S. collected and curated the CRO data. S.M. guided the contour selection for the PMH cohort. S.M., S.C., M.N. and C.B. provided expert knowledge for clinical questions. K.P. provided the computational framework and critically reviewed the methodological approach. All authors read and approved the manuscript.

\section{Funding}

Open Access funding enabled and organized by Projekt DEAL.

\section{Competing interests}

The authors declare no competing interests.

\section{Additional information}

Supplementary Information The online version contains supplementary material available at https://doi.org/ 10.1038/s41598-021-85671-y.

Correspondence and requests for materials should be addressed to G.L.

Reprints and permissions information is available at www.nature.com/reprints.

Publisher's note Springer Nature remains neutral with regard to jurisdictional claims in published maps and institutional affiliations. 
(c) (i) Open Access This article is licensed under a Creative Commons Attribution 4.0 International cc) License, which permits use, sharing, adaptation, distribution and reproduction in any medium or format, as long as you give appropriate credit to the original author(s) and the source, provide a link to the Creative Commons licence, and indicate if changes were made. The images or other third party material in this article are included in the article's Creative Commons licence, unless indicated otherwise in a credit line to the material. If material is not included in the article's Creative Commons licence and your intended use is not permitted by statutory regulation or exceeds the permitted use, you will need to obtain permission directly from the copyright holder. To view a copy of this licence, visit http://creativecommons.org/licenses/by/4.0/.

(C) The Author(s) 2021 